\title{
Valores hematológicos de capivaras (Hydrochoerus hydrochaeris) Rodentia:Hydrochoeridae) de vida livre na região de Campinas-SP
}

\author{
Capybara's (Hydrochoerus hydrochaeris) hematological values \\ free ranging life from Campinas regions, \\ São Paulo state, Brazil \\ Daniela Alves Madella ${ }^{{ }^{*}}$ Elias José Rodrigues Neto ${ }^{1}$ \\ Maria Elisa Felisberto ${ }^{1}$ Celso Eduardo de Souza ${ }^{1}$
}

\begin{abstract}
O objetivo deste estudo foi determinar os valores RESUMO hematológicos de 14 capivaras de vida livre capturadas nos municípios de Campinas, Monte Alegre do Sul e de São João da Boa Vista. Para a obtenção das amostras de sangue, os animais foram anestesiados via intramuscular, mediante associação de cloridrato de xilazina $2 \%$ e cloridrato de ketamina $1 \%$, em doses de $4,5 \mathrm{mg} / \mathrm{kg} / \mathrm{pv}$ e $5,0 \mathrm{mg} / \mathrm{kg} / \mathrm{pv}$, respectivamente. $O$ sangue foi coletado em tubos contendo EDTA. Para a determinação dos números de eritrócitos e leucócitos, foi utilizada a metodologia tradicional da câmara de Neubauer. O hematócrito foi determinado pelo método de microhematócrito e a hemoglobina pelo método da cianometahemoglobina. Para a contagem diferencial de leucócitos, foi utilizada a técnica de Shilling. Os índices hematimétricos (VCM, HCM E CHCM) foram determinados com os valores encontrados na série vermelha. Os resultados da série eritrocitária foram: $4,5 \times 10^{6} / \mu \mathrm{L} \pm 0,2$; hemoglobina: 13.5g/dL \pm 0,7; hematócrito: 40,4\% \pm 2 ; VCM: 90,2 fL $\pm 0,9$; HCM: $30,1 \mathrm{pg} \pm 0,7 ;$ CHCM: $33,3 \mathrm{~g} / \mathrm{dL} \pm 1$. Na série leucocitária: $5,3 \times 10^{3} / \mu \mathrm{L} \pm 2$; bastonetes: $1 \% \pm 2,1$; segmentados: $36,6 \% \pm 19,9$; eosinófilos: $3,5 \% \pm 11,7$; basófilos: 0\%; linfócitos: $58,8 \% \pm 19,6$; monócitos: $1,5 \% \pm$ 2,1. Não foram encontrados os mielócitos e metamielócitos.
\end{abstract}

Palavras-chave: capivara, Hydrochoerus hydrochaeris, hematologia, hemograma.

\section{ABSTRACT}

The purpose of this study was to determine the hematological values of fourteen free life capybaras captured in three cities of São Paulo State, Brazil (Campinas, Monte
Alegre do Sul and São João da Boa Vista). Blood samples were obtained with the animals anesthetized through association of $2 \%$ xilazine chloridrate and $1 \%$ ketamine chloridrate, in the doses of $4.5 \mathrm{mg} / \mathrm{kg} / \mathrm{pv}$ and $5.0 \mathrm{mg} / \mathrm{kg} / \mathrm{pv}$ respectively. The blood was collected using tubes containing EDTA. To determine the number of erythrocytes and leukocytes was used the traditional methodology of the Neubauer chamber. The hematocrit was determined by the microhematocrit method and the hemoglobin by the cianometahemoglobin. For leukocyte differential count the Shilling's method was used. The hematimetric (MCV, $M C H$ and $M C H C$ ) indexes were determined with the values found in the red serie. The results were: erythrocytes: $4.5 \times 10^{6} / \mu \mathrm{l} \pm 0.2$; hemoglobin: $13.5 \mathrm{~g} / \mathrm{dl} \pm 0.7$; hematocrit: $40.4 \% \pm 2$; $\mathrm{MCV}$ : 90.2fl \pm 0.9; $\mathrm{MCH}: 30.1 \mathrm{pg} \pm 0.7$; MCHC: $33.3 \mathrm{~g} / \mathrm{dl} \pm 1$. In the leukocytes series: $5.3 \times 10^{3} / \mu \mathrm{l} \pm 2$; band neutrophil:1 $\% \pm 2.1$; segmenter: $36.6 \% \pm 19.9$; eosinophils: $3.5 \% \pm 11.7$; basophiles: 0\%; lymphocytes: $58.8 \% \pm 19.6$; monocytes: $1.5 \% \pm 2.1$. Myelocytes and metamyelocytes were not found.

Key words: capybara, Hydrochoerus hydrochaeris, hematology, hemogram.

A hematologia dos animais silvestres, especialmente os da fauna brasileira, ainda é um campo de trabalho científico pouquíssimo explorado, sendo necessários estudos exaustivos para que se possa chegar a um nível adequado de compreensão de suas particularidades.

Para o campo da Medicina Veterinária a determinação dos valores hematológicos normais para

\footnotetext{
${ }^{1}$ Superintendência de Controle de Endemias (SUCEN), Laboratório de Bioecologia, Epidemiologia e Controle de Carrapatos de Importância Médica (LBECCIM). Rua São Francisco, n. 630, 13871-118, São João da Boa Vista, SP, Brasil. E-mail: labcarrapatos@sucen.sp.gov.br e danielamadella@bol.com.br.*Autor para correspondência.
} 
a capivara é necessária, a fim de interpretar as alterações nos diversos quadros clínicos. A análise sangüínea é uma importante ajuda para oferecer o conhecimento e o modo de resposta do tecido hematopoético do animal, permitindo o diagnóstico das doenças hematológicas e a sua recuperação sobre os elementos constituintes do sangue, proporcionando dados sobre o grau de reação do organismo frente à presença de alguns microorganismos invasores, de deficiências nutricionais, etc (GUERCI, 1985).

A capivara é a maior espécie de roedor conhecida. Pertence à subordem Caviomorphae, família Hydrochoridae e subfamília Cavioidae. O gênero Hydrochoerus possui quatro espécies: Hydrochoerus hydrochaeris e $\boldsymbol{H}$. isthmius, $\boldsymbol{H}$. dabbnei e $\boldsymbol{H}$. uruguayenses. Mede aproximadamente entre 1 e $1,5 \mathrm{~m}$ de comprimento, 0,5 a 0,65m de altura e, no Brasil, o peso corpóreo ultrapassa $80 \mathrm{~kg}$. Os olhos, os orifícios nasais, como também os dedos, que são todos unidos entre si por uma membrana, são adaptados à vida aquática. Apresenta uma glândula sebácea de cor escura na parte superior da cabeça, na qual existem numerosas células secretoras de um líquido branco e pegajoso que serve para demarcar o território do grupo familiar (ALHO, 1986; ALHO et al., 1987). A capivara é o único roedor que possui em sua pele glândulas sudoríparas (PEREIRA et al., 1980).

A hematologia desta espécie ainda é pouco estudada. A maioria dos poucos relatos existentes na literatura é com animais em cativeiro com finalidade comercial. AROUCA et al. (2000) estudaram animais em cativeiro no município de Botucatu-SP; VAN DEN HEIJDEN (2000) verificou a hematologia de capivaras do zoológico de São Bernardo do Campo-SP e de criadouros comerciais de sete municípios paulistas e de um gaúcho, observando alterações hematológicas decorrentes do parasitismo por carrapatos; e MUÑOZ \& MONTOYA(2001) estudaram animais em cativeiro na Amazônia Peruana.

A capivara possui eritrócito muito grande, com diâmetro de 8,5 a 9,0 (AROUCA et al., 2000). Os neutrófilos são também denominados de pseudoeosinófilos, pois apresentam grânulos eosinofílicos bem marcantes no citoplasma e núcleo com lóbulos distintos. A diferenciação destes com o verdadeiro eosinófilo dá-se pelo tamanho e distribuição dos grânulos dos pseudoeosinófilos (ARCHER \& JEFFCOTT, 1977; HAWKEY \& DENNET, 1989; JAIN, 1993). Os basófilos, monócitos e linfócitos da capivara assemelham-se aos de outras espécies animais. Eventualmente, nos linfócitos, observam-se inclusões citoplasmáticas conhecidas como Corpúsculos de Kurloff (JAIN, 1986; HAWKEY \& DENNET., 1989).
O objetivo deste trabalho foi determinar os valores hematológicos de 14 capivaras de vida livre, sendo 4 machos e 10 fêmeas, cuja idade não foi possível determinar, contribuindo para o conhecimento das características hematológicas desta espécie. Este trabalho foi realizado no período de janeiro a abril de 2004, com animais do Lago do Café, em Campinas-SP, da Fazenda do Instituto Agronômico de Campinas, em Monte Alegre do Sul-SP, e da mata ciliar do Rio Jaguarimirim, em São João da Boa Vista-SP.

Os animais foram capturados utilizando-se bretes móveis e ceva constituída de capim napier, milho e sal mineral. Após o jejum de doze horas, foram anestesiados, por via intramuscular, com associação de cloridrato de xilazina $2 \%$ ( $4 \mathrm{mg} / \mathrm{kg} / \mathrm{pv}$ ) e cloridrato de ketamina $1 \%(5 \mathrm{mg} / \mathrm{kg} / \mathrm{pv})$. Através de punção da veia femural, foram coletados três mililitros de sangue em tubos contendo anticoagulante EDTA na proporção de $1 \mathrm{mg} / \mathrm{mL}$. Cada animal foi pesado e identificado com um "microchip". As amostras foram processadas no Laboratório da Superintendência de Controle de Endemias de São João da Boa Vista - SP. A metodologia para a determinação dos valores hematológicos seguiuse à descrição de BIRGEL \& BENESI (1982).

Para a determinação do número de hemácias e leucócitos por mililitro de sangue, utilizou-se a câmara de Neubauer, com diluição em pipeta de Thomas. O líquido de Hayem foi empregado como diluente para contagem dos eritrócitos na diluição de 1:200, contando-se os eritrócitos nos cinco quadrados secundários do quadrado central e multiplicando-os por 10000. Para os leucócitos, diluiu-se o sangue com o líquido de Türk na proporção de 1:20 e contou-se os leucócitos nos quatro quadrados encontrados nos extremos da câmara. Nas duas técnicas citadas acima, antes das contagens das células, a câmara de Neubauer permaneceu por cinco minutos dentro de uma placa de Petri invertida, contendo um chumaço de algodão úmido, permitindo assim uma perfeita sedimentação das células. Para a determinação do hematócrito, o tubo de microhematócrito foi preenchido com sangue em aproximadamente $3 / 4$ de sua capacidade e vedado em uma das extremidades com auxílio do bico de Bunsen. Colocou-se o capilar na microcentrífuga por cinco minutos, a 3000rpm, realizando-se a leitura no cartão específico. Adosagem de hemoglobina foi determinada adicionando-se $5 \mathrm{ml}$ do líquido de Drabkin em três tubos de vidro, identificando como respectivamente branco (B), padrão (P) e amostra (A). Adicionou-se $20 \mu \mathrm{L}$ de padrão de hemoglobina no tubo P e $20 \mu \mathrm{L}$ da amostra de sangue no tubo A, homogeneizou-se-a e fez-se a leitura em espectrofotômetro a 540nm. Para a observação da morfologia das células vermelhas e contagem 
diferencial das células brancas, fez-se um esfregaço de sangue em lâmina, que foi fixada em álcool metílico por 30 minutos e corada através da técnica de Giemsa. Depois de lavada e secada à temperatura ambiente, examinou-se a lâmina no microscópio óptico. Foram contadas 100 células seguindo a técnica de zig-zag de Shilling.

Os índices hematimétricos foram calculados utilizando-se os resultados determinados na série vermelha. O volume corpuscular médio (VCM) foi em fentolitros, a hemoglobina corpuscular média (HCM) em picogramas e a concentração de hemoglobina corpuscular média (CHCM) em g/dL.

No presente estudo, foram obtidos os valores médios da série eritrocitária apresentados na tabela 1, e os valores médios da série leucocitária na tabela 2.

O valor médio para eritrócitos foi superior ao reportado por FOWLER (1986), AROUCA et al. (2000), VANDEN HEIJDEN(2000), FOWLER \& CUBAS (2001) e MUÑOZ \& MONTOYA(2001). Provavelmente, a variação dos eritrócitos ocorre em função da metodologia utilizada, dos fatores ambientais (altitude e clima), do estresse pela captura, do tipo de criação (intensiva e extensiva) e da alimentação (JAIN, 1986).

O valor da hemoglobina média obtida neste estudo foi superior à descrita por FOWLER (1986) e FOWLER \& CUBAS (2001). Porém, inferior aos valores reportados por AROUCA et al. (2000), VAN DEN HEIJDEN (2000), e MUÑOZ \& MONTOYA (2001). Esta diferença observada pode ser devida ao tipo de alimentação ou às técnicas utilizadas, tais como: hematina ácida, oxihemoglobina e a cianometahemoglobina, com margem de erro de 15\%, $10 \%$ e de 1 a $\%$, respectivamente (MEDWAY, 1973; BUSH, 1982; MAXIMINE, 1991).

O valor médio obtido para o hematócrito foi inferior às médias encontradas por AROUCA et al. (2000) e superior às médias encontradas por FOWLER (1986), VANDEN HEIJDEN (2000), FOWLER \& CUBAS (2001) e MUÑOZ \& MONTOYA (2001), provalmente em

Tabela 1 - Valores obtidos da série eritrocitária de 14 capivaras de vida livre na região de Campinas-SP.

\begin{tabular}{ll}
\hline Eritrograma & Média \pm DP \\
\hline Eritrócitos $\left(\mathrm{x} 10^{6} / \mu \mathrm{L}\right)$ & $4,5 \pm 0,2$ \\
Hemoglobina $(\mathrm{g} / \mathrm{dL})$ & $13,5 \pm 0,5$ \\
Hematócrito $(\%)$ & $40,4 \pm 2$ \\
VCM (fL) & $90,2 \pm 1$ \\
HCM (pg) & $30,1 \pm 0,7$ \\
CHCM (g/dL) & $33,3 \pm 1$ \\
\hline
\end{tabular}

Tabela 2 - Valores obtidos da série leucocitária de 14 capivaras de vida livre na região de Campinas-SP.

\begin{tabular}{ll}
\hline Leucograma & Média \pm DP \\
\hline Leucócitos $\left(\times 10^{3} / \mu \mathrm{L}\right)$ & $5,3 \pm 2$ \\
Bastonetes (\%) & $1 \pm 2,1$ \\
Neutrófilos (\%) & $36,6 \pm 19,9$ \\
Eosinófilos (\%) & $3,5 \pm 11,7$ \\
Basófilos (\%) & 0 \\
Linfócitos (\%) & $58,9 \pm 19,6$ \\
Monócitos (\%) & $1,5 \pm 2,1$ \\
\hline
\end{tabular}

virtude da técnica utilizada na determinação do hematócrito, da diferença de altitude e/ou do manejo dos animais. As médias obtidas para o VCM são inferiores às apresentadas por FOWLER (1986), AROUCA et al. (2000), VAN DEN HEIJDEN (2000), MUÑOZ \& MONTOYA(2001) e FOWLER \& CUBAS (2001). A média do CHCM apresentou-se inferior às apresentadas por MUÑOZ \& MONTOYA(2001) e VAN DEN HEIJDEN (2000), com relação aos animais parasitados por carrapatos.

Os valores de leucócitos totais foram inferiores aos reportados por FOWLER (1986), FOWLER \& CUBAS (2001) e MUÑOZ \& MONTOYA (2001). Porém, superiores aos encontrados por AROUCA et al. (2000). Isto pode ser devido ao número diferente de leucócitos ser significativamente influenciado pelo local da punção sangüínea, pela idade do animal e pela atividade muscular (JAIN, 1986).

Para os neutrófilos, o valor médio encontrado foi inferior aos valores relatados por FOWLER (1986), AROUCA et al. (2000), FOWLER \& CUBAS (2001) e MUÑOZ \& MONTOYA(2001). Para os linfócitos, o valor médio foi bem superior aos valores apresentados por FOWLER (1986), AROUCA et al. (2000), VAN DEN HEIJDEN (2000), MUÑOZ \& MONTOYA (2001) e FOWLER \& CUBAS (2001), provavelmente em virtude do estresse causado pela captura. O valor médio dos eosinófilos foi semelhante ao encontrado por AROUCA et al. (2000), superior ao valor descrito por MUÑOZ \& MONTOYA (2001) e inferior ao encontrado por VAN DEN HEIJDEN (2000). O valor médio de monócitos foi bem inferior ao relatado por FOWLER (1986), AROUCA et al. (2000), VAN DEN HEIJDEN(2000), FOWLER \& CUBAS (2001) e MUÑOZ \& MONTOYA (2001). Não foram encontrados basófilos, concordando com MUÑOZ \& MONTOYA (2001). Contudo, FOWLER (1986), AROUCA et al. (2000), VAN DEN HEIJDEN (2000) e FOWLER \& CUBAS (2001) relataram valores para basófilos. 
Possivelmente, as diferenças entre os valores obtidos neste estudo e os existentes na literatura ocorrem em virtude de fatores climáticos, da variação de altitude e das técnicas de manejo e criação dos animais (JAIN, 1986). As capivaras deste estudo são de vida livre. Além de sofrerem o estresse causado pela captura, têm uma maior tendência de exposição a antígenos ambientais, devido à ausência de manejos zootécnico e sanitário, podendo desenvolver um sistema imunológico particular para a defesa do organismo frente às diversas situações.

Diante dos resultados obtidos, conclui-se que são necessários maiores estudos para determinar os valores normais para o hemograma de capivaras de vida livre.

\section{REFERÊNCIAS}

ALHO, C.J.R. Criação e manejo de capivaras em pequenas propriedades rurais. Brasília: Embrapa, 1986. 39p.

ALHO, C.J.R. et al. Ecologia de capivaras do Pantanal: - I Habitats, densidades e tamanho do grupo. Rev Brasil Biol, v.47, n.1/2, p.87-97, 1987.

ARCHER, R.K.; JEFFCOTT, L.B. Comparative clinical haematology. Austrália: Blackwell Scientific Publications, 1977. 737p.

AROUCA, M.E. et al. Valores hematológicos de capivaras criadas no município de Botucatu, SP. Ciência Rural, Santa Maria, v.30, n.5, p.813-817, 2000.

BIRGEL, E.H.; BENESI, F.H. Patologia clínica veterinária. São Paulo: Sociedade Paulista de Medicina Veterinária, 1982. 346p.

BUSH, B.M. Manual de laboratório veterinário de análises clínicas. Zaragoza: Acribia, 1982. 467p.
FOWLER, M.E. Zoo and wild animal medicine. 2.ed. Philadelphia: Saunders, 1986. 1127p.

FOWLER, M.E.; CUBAS, Z.S. Biology, medicine, and surgery of South American Wild Animals. Iowa: Iowa State University, 2001. 536p.

GUERCI, A. Métodos de análisis clínicos y su interpretación. Buenos Aires: El Ateneo, 1985. 513p.

HAWKEY, C.M.; DENNET, T.B. Comparative veterinary haematology. Ipswich : W.S.Cowell, 1989. 192p.

JAIN, N.C. Essentials of veterinary hematology. Philadelphia: Lea \& Febiger, 1993. 417p.

JAIN, N.C. Schalm's veterinary hematology. 4.ed. Philadelphia: Lea \& Febiger, 1986. 1221p.

MÁXIMINE, M. Manual de patología clínica en veterinaria. México: Limusa, 1991. 389p.

MEDWAY, M. Patología clínica veterinária. México: Centro Regional de Ayuda Técnica, 1973. 253p.

MUÑOZ, K.D.; MONTOYA, E.G. Valores hemáticos del roncoso (Hydrochaeris hydrochaeris) en cautiveiro en la Amazonía Peruana. Capturado em 17 ago. 2004. Online. Disponível na Internet http://sisbib.unmsm.edu.pe/BVRevistas/ veterinaria/Vol12_N1_2001/val_hema_ronso.htm.

PEREIRA, $\mathrm{N}$ et al. The structure of the skin of the capybara. Acta Cient Vzlana, v.31, p.361-364, 1980.

VAN DEN HEIJDEN, K.M. Carrapatos em capivaras (Hydrochoerus hydrochaeris) mantidas em cativeiro: espécies envolvidas e reação celular elicitada no hospedeiro. 2000. 86f. Dissertação (Mestrado em Patologia Experimental e Comparada) - Curso de Pós-graduação em Patologia, Faculdade de Medicina Veterinária e Zootecnia da Universidade de São Paulo. 